Complete resolution of symptoms, or adequate resolution that required no further treatment, was achieved in 13 patients, and 4 patients had satisfactory symptom relief following a second treatment session. Symptoms failed to resolve adequately in 3 patients. No sedation was required during treatment sessions, and no adverse events were recorded.

The authors conclude that small-volume formalin instillation is a simple, safe, rapid, and effective treatment for rectal bleeding in patients with radiation proctopathy. Prospective trials are now required, to determine whether small-volume formalin instillation should become the first-line therapy for hemorrhagic radiation proctopathy.

Original article Cullen SN et al. (2006) Treatment of haemorrhagic radiation-induced proctopathy using small volume topical formalin instillation. Aliment Pharmacol Ther 23: $1575-1579$

\section{Testosterone therapy rebuilds muscle in older men}

Testosterone is a potential treatment for physical dysfunction due to age-related decreases in muscle mass. These decreases are largely the result of a loss of type II skeletal muscle fibers, and testosterone therapy has been shown to increase muscle mass. A group of US investigators has found that administration of exogenous testosterone results in dose-dependent increase in the number of both type I and type II fibers.

Endogenous testosterone production was blocked in 36 healthy men (aged 60-75 years), who subsequently received testosterone enanthate injections $(25 \mathrm{mg}, 50 \mathrm{mg}, 125 \mathrm{mg}, 300 \mathrm{mg}$, or $600 \mathrm{mg}$ ) weekly for 20 weeks. Analysis of cross-sectional biopsies of the participants' thigh muscles taken before and after treatment showed that the number of type I and type II fibers increased dose-dependently. The number of myonuclei and satellite cells also increased dose-dependently (only with the three highest doses); this finding suggests that testosterone promotes entry of myogenic precursor cells into the cell cycle. Further analysis showed that the increased number of satellite cells was the result of satellite-cell replication and activation.

Although these results are highly encouraging, the use of high doses of testosterone to rebuild skeletal muscle in older men is limited by adverse effects. The authors call for further study of the mechanisms involved, in the hope that new targets for anabolic therapy will be discovered.

Original article Sinha-Hikim I et al. (2006) Effects of testosterone supplementation on skeletal muscle fiber hypertrophy and satellite cells in community dwelling, older men. J Clin Endocrinol Metab [doi: 10.1210/jc.2006-0357]

\section{Less postoperative pain with laparoscopic than with open donor nephrectomy}

Kidney transplant donors who undergo laparoscopic surgery have significantly less postoperative pain and more-rapid convalescence than those who undergo traditional open surgery, according to the results of a single-center, prospective, controlled trial in Norway. A few previous studies have had similar findings, but the larger sample size of the present investigation could make it the most accurate to date.

The donors were randomized to laparoscopic $(n=63)$ or open $(n=59)$ donor nephrectomy. On the second postoperative day, self-reported pain scores were not significantly different between the two groups, but 1 month after donation, mean pain scores measured on numeric rating scales were significantly lower in the laparoscopy group than in the open-surgery group $(P=0.002)$. At 1 month post-donation, more open-surgery donors than laparoscopic donors had used pain medication in the previous $24 \mathrm{~h}$ (24.6\% vs $11.3 \%$; $P=0.048)$. In addition, in multiple regression analyses, donors who underwent laparoscopic surgery had a shorter period of sick leave than those who underwent open surgery $(P=0.011)$. Major postoperative complications, including jejunal perforation, occurred in five patients in the laparoscopic group. There were no major complications in patients in the open-surgery group.

Laparoscopy involves creating a small incision through which the kidney is removed, minimizing tissue damage; the donor should, therefore, recover faster and return to work sooner than after open surgery. As postoperative pain and disruption to life are disincentives to potential living donors, offering the laparoscopic option might increase donation rates.

Original article Andersen MH et al. (2006) Postoperative pain and convalescence in living kidney donors-laparoscopic versus open donor nephrectomy: a randomized study. Am J

Transplant 6: 1438-1443 